\title{
Guidelines on Setting Up Stations for Remote Viewing of Nuclear Medicine and Molecular Imaging Studies During COVID-19
}

\author{
Jerold W. Wallis ${ }^{1}$, Ran Klein ${ }^{2}$, Tyler J. Bradshaw ${ }^{3}$, Ciprian Catana ${ }^{4}$, Mathieu Hatt ${ }^{5}$, Richard Laforest ${ }^{1}$, Chi Liu ${ }^{6}$, \\ Osama Mawlawi $^{7}$, Keisha C. McCall ${ }^{8}$, Dustin R. Osborne, DABSNM ${ }^{9}$, Jing Tang, DABSNM ${ }^{10}$, R. Glenn Wells, \\ FCCPM $^{11}$, and Munir Ghesani, FACNM, FACR ${ }^{12}$

\begin{abstract}
${ }^{1}$ Mallinckrodt Institute of Radiology, Washington University School of Medicine, St. Louis, Missouri; ${ }^{2}$ Division of Nuclear Medicine, Department of Medicine, University of Ottawa, Ottawa, Ontario, Canada; ${ }^{3}$ Department of Radiology, University of Wisconsin, Madison, Wisconsin; ${ }^{4}$ Athinoula A. Martinos Center for Biomedical Imaging, Department of Radiology, Massachusetts General Hospital and Harvard Medical School, Charlestown, Massachusetts; ${ }^{5}$ LaTIM, INSERM, UMR 1101, University of Brest, Brest, France; ${ }^{6}$ Department of Radiology and Biomedical Imaging, Yale University, New Haven, Connecticut; ${ }^{7}$ Department of Imaging Physics, M.D. Anderson Cancer Center, Houston, Texas; ${ }^{8}$ Department of Radiology, Henry Ford Health System, Detroit, Michigan; ${ }^{9}$ Molecular Imaging and Translational Research Program, University of Tennessee Graduate School of Medicine, Knoxville, Tennessee; ${ }^{10}$ Oakland University, Rochester, Michigan; ${ }^{11}$ Department of Cardiology, University of Ottawa Heart Institute, Ottawa, Ontario, Canada; and ${ }^{12}$ Icahn School of Medicine at Mount Sinai, New York, New York
\end{abstract}

CE credit: For CE credit, you can access the test for this article, as well as additional JNMT CE tests, online at https://www.snmmilearningcenter.org. Complete the test online no later than March 2024. Your online test will be scored immediately. You may make 3 attempts to pass the test and must answer $80 \%$ of the questions correctly to receive $1.0 \mathrm{CEH}$ (Continuing Education Hour) credit. SNMMI members will have their CEH credit added to their VOICE transcript automatically; nonmembers will be able to print out a CE certificate upon successfully completing the test. The online test is free to SNMMI members; nonmembers must pay $\$ 15.00$ by credit card when logging onto the website to take the test.

The current pandemic has created a situation where nuclear medicine practitioners and medical physicists read or process nuclear medicine images remotely from their home office. This article presents recommendations on the components and specifications when setting up a remote viewing station for nuclear medicine imaging.

Key Words: Physics, Instrumentation, and Data Sciences Council (PIDSC); remote viewing; guidelines; COVID-19

J Nucl Med Technol 2021; 49:2-6

DOI: $10.2967 /$ jnmt.120.261890

$\mathbf{T}$ he coronavirus disease 2019 (COVID-19) pandemic has created new realities, with an increasing number of nuclear medicine physicians having to read remotely from their homes to comply with social distancing mandates. Several recent publications have described specifications and tips on how to set up viewing stations to allow reading from home (1-4), but none of these focuses on nuclear medicine imaging. In this article, the Physics, Instrumenta-

Received Dec. 29, 2020; accepted Dec. 29, 2020.

For correspondence contact: Jerold W. Wallis, Mallinckrodt Institute of Radiology, 510 S. Kingshighway Blvd., St. Lois, MO 63110.

E-mail: wallisj@mir.wustl.edu

Published online Dec. 30, 2020.

COPYRIGHT (C) 2021 by the Society of Nuclear Medicine and Molecular Imaging. tion, and Data Sciences Council of the Society of Nuclear Medicine and Molecular Imaging (SNMMI) responds to a request from the SNMMI COVID-19 task force that guidelines be provided on setting up a remote viewing station that is specific to nuclear medicine imaging. The article expands on previous guidance from the SNMMI on remote nuclear medicine viewing (5).

If you are setting up a PACS station in your home office using equipment identical to that in your work office, follow your work guidelines on which hardware to purchase. Alternatively, if you are setting up your own remote viewing station with your own hardware, consider the guidelines detailed in this article.

\section{ERGONOMICS}

An ergonomic working environment is highly desirable for nuclear medicine reading stations. For example, chair and desk height, monitor height and adjustability, placement of the mouse, and possible use of a footrest should all be taken into consideration when creating a home working environment. Likewise, good air circulation and temperature and a quiet (few distractions) room are essential to sustained comfort and alertness over prolonged working hours (4).

\section{COMPUTER HARDWARE}

The level of computer performance required will depend on the applications to be run. Two alternative application configurations must be considered: a thick client and a thin client. 


\section{Thick Client}

A thick client is when the image visualization and analysis software are installed on your computer and the image data are transferred to it from the image archive server (e.g., PACS). In this configuration, your computer performs the image processing and rendering and therefore requires more memory and processing power. Data transfer to your organization occurs only when downloading image data to review or when sending back secondary screen captures, reports, or other data. Patientsensitive data will reside locally, and therefore your computer should be secured according to your hospital's policy, including hard-drive encryption, malware protection, and restrictions on applications that can be installed and websites that may be accessed. In some cases, your institutional information technology department or software vendor will require you to use computer hardware procured through them.

\section{Thin Client}

A thin client is when the image processing and rendering are performed on a centralized server to which your

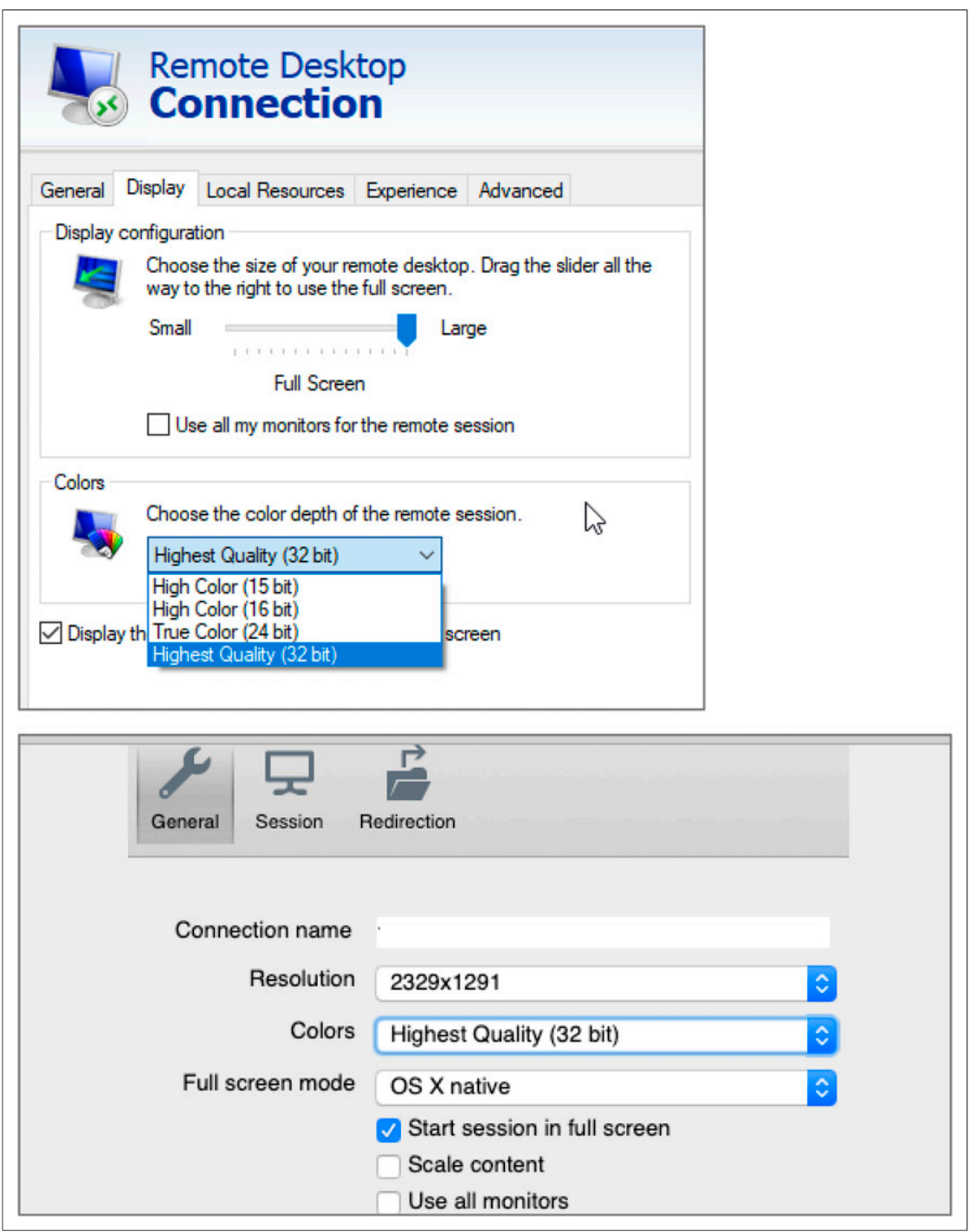

FIGURE 1. Color-depth choices in RDP settings for Windows (top) and Mac (bottom). computer connects using a special client (e.g., Citrix or remote desktop protocol [RDP]). In this case, your computer only mirrors the image generated by the server and sends back keyboard and mouse commands. In this configuration, your computer requires relatively little processing power, which even a mid-level modern laptop can provide. Network traffic is persistent throughout the viewing session, requiring sufficient bandwidth and a stable connection. Patient-sensitive data may not actually reside on your computer, and security requirements may therefore be lax, even to the point where your personal home computer may be used.

Because a complete reporting workstation will require multiple software applications, both thin- and thick-client applications may reside on the computer. Regardless, a dedicated graphics card is recommended and may be required by some applications. The computer operating system may be dictated by applications that must run on the computer (e.g., viewer, electronic medical records, and reporting software). It is recommended that you consult with both your institutional information technology department and software vendors for specific technical requirements before acquiring a computer. The computer specifications should match or exceed those posed by the most demanding application.

\section{SECURITY}

Your hospital should provide secure access via a cloud-based image viewing service, a virtual private network to the hospital network, or an RDP to your office personal computer. These may require installation of software on your home computer and authentication apps on your phone.

\section{AMBIENT LIGHT}

To reduce eye strain and optimize image viewing, ambient lighting must be configured to eliminate glare and allow the user to view the full gray-scale range of the displayed images. Use of dimmable lighting reflecting off the wall behind the computer monitors is an effective, inexpensive, and highly configurable solution and might be considered. In a nuclear medicine reading room, ambient light should be just bright enough to allow a small-text paper document (e.g., a newspaper) to be read.

Ambient light can be measured with a light reader and, if measured, should be in the range of 25-50 lux in intensity (4). An Android phone app has also been used to measure ambient light in 
radiology reading rooms (6) but has not been validated across all models of phones.

\section{MONITORS}

\section{Size}

The monitors must meet minimum pixel size and physical size requirements for the software you will be using. Use of multiple applications (for viewing patient charts, dictating, and image viewing) will likely require more than one monitor; 24-in (61-cm) $1,920 \times 1,200$ or better monitors with color capability will likely be sufficient for most applications. This specification corresponds to 94.3 pixels per inch (0.269-mm dot pitch). Some users may prefer a higher pixel density, but it is unlikely to enhance nuclear medicine image interpretation. A single large monitor may be a substitute for multiple monitors and has the advantage of alleviating the need to cross-calibrate monitors.

\section{Consumer-Grade Versus DICOM}

Most institutions use high-end consumer-grade color monitors rather than medical gray-scale DICOM monitors for reading of PET/CT, PET/MRI, and other nuclear medicine studies. Similar monitors may therefore be used at home, provided they meet gray-scale display specifications and are calibrated. Special consideration should be given if the at-home nuclear medicine physician is formally reading other modalities such as mammography, which has more stringent display requirements.

\section{Display Bit Depth}

If a remote desktop is used, the quality of the RDP connection display must be set to the highest quality (32-bit) in the connection settings (Fig. 1). Use of lower bit depths may limit your gray scale to only 32 gray shades (or less). Such lower bit depths may speed access for word processing activities but are not adequate for nuclear medicine use. (Recent versions of RDP default to a 32-bit depth, and some enterprise settings may force a 32-bit depth regardless of user selections.)

Similarly, on your home computer the display quality should be set to the maximum number of colors. Modern operating systems and display cards now typically default to 32-bit depth and may no longer allow setting to lower (inadequate) bit depths. Bit depth can be visually assessed using the gray-sphere test pattern available online $(5,9)$. If you see a smooth gray range, you have the desired bit depth setting (Fig. 2). If you see circular bands in the gray sphere, you have an inadequate bit depth setting.

\section{Display Brightness}

No strict guidelines for display brightness are available, but $300 \mathrm{~cd} / \mathrm{m}^{2}$ (nits) is regarded as a reasonable minimum specification when purchasing a monitor. This level of brightness will allow enough excess range to permit for some dimming during monitor aging and adjustments during gray-scale calibration. Equally or more important is the ability to darken the room in which the interpretation is being done, since perceived display brightness and room brightness are interrelated.

If measured, display brightness should be at least $150 \mathrm{~cd} / \mathrm{m}^{2}$ (nits) after monitor calibration, as measured by a dedicated

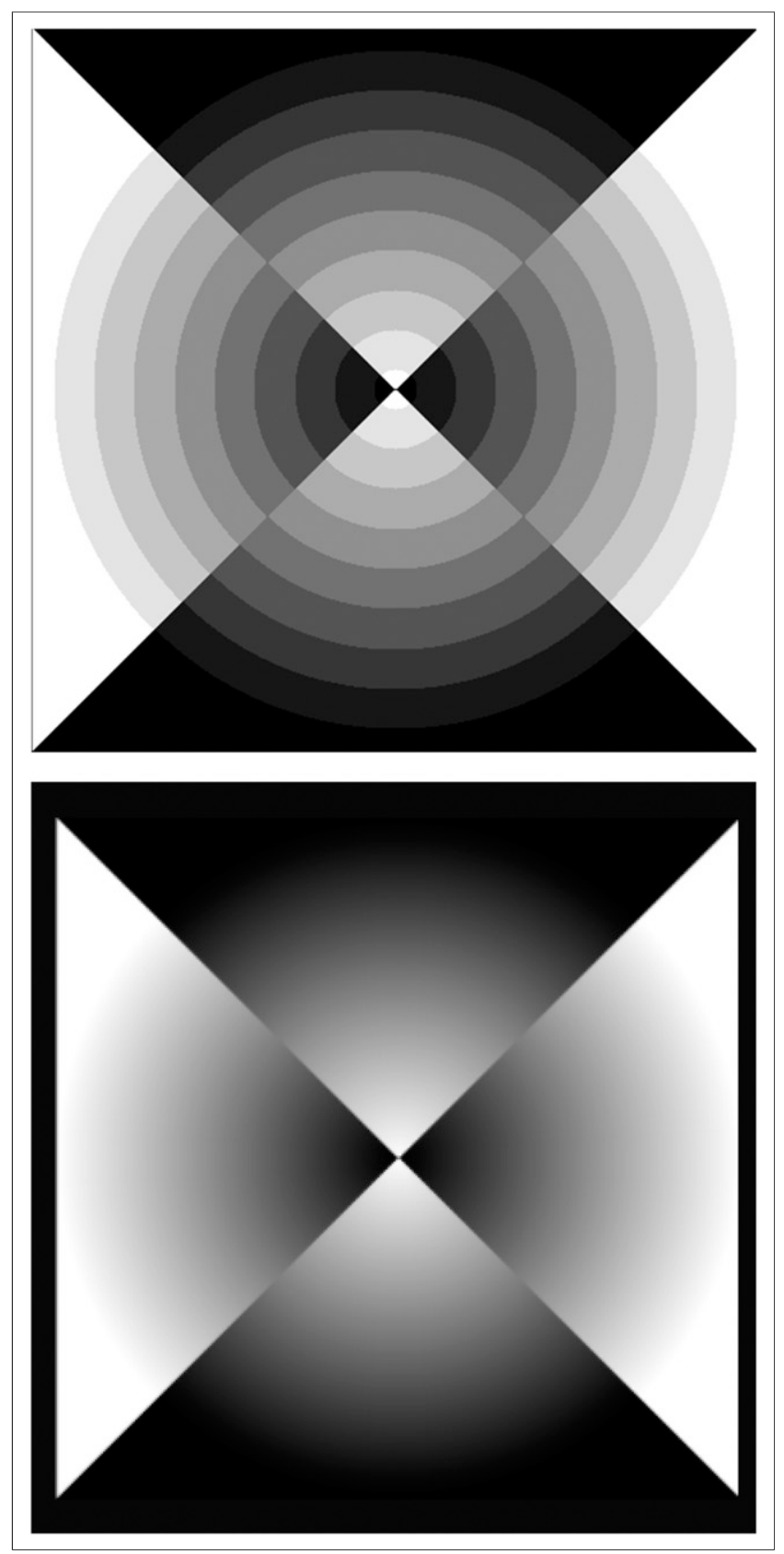

FIGURE 2. (Bottom) Image created with smooth gray-scale range, which should not have visible rings. (Top) Image demonstrating rings that might be seen if display depth is not properly set. (Courtesy of J. Anthony Parker, MD, Beth Israel Deaconess Medical Center, Boston, MA.)

light meter. It has been suggested that smartphone applications might be used for this purpose, but they have not been adequately validated against true light meters $(7,8)$.

\section{Gray-Scale Range}

The monitor should be able to be calibrated to display the small $5 \%$ and $95 \%$ boxes within the larger boxes of the Society of Motion Picture and Television Engineers (SMPTE) test pattern in the lighting conditions under which you will be reading (Fig. 3). Links to these gray-sphere and 


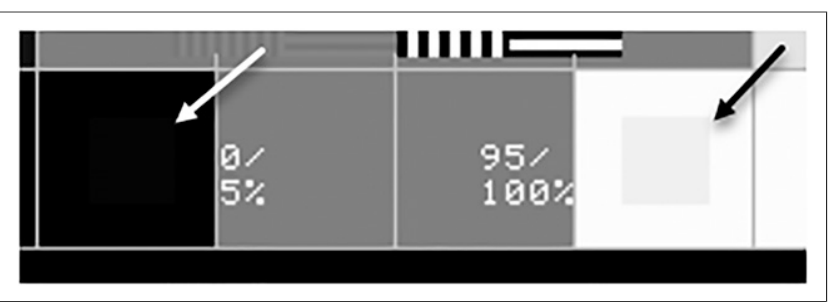

FIGURE 3. Small portion of SMPTE pattern, with arrows indicating small $5 \%$ and $95 \%$ boxes that should be visible after monitor calibration. Best viewed in full test pattern (see Table 1).

SMPTE test patterns are available online ( 9 , also see Table 1$)$. Calibration should be performed using the monitor brightness and contrast controls on installation of the monitor and periodically at 6- to 12-mo intervals or when major hardware and software components have been replaced or modified. If the monitor has a third, black-level, control, this will greatly facilitate calibration and make it much more likely that the monitor can be calibrated adequately for nuclear medicine use. Liquidcrystal-display monitors using the in-plane-switching (IPS) technology are more likely to display the full grayscale range than are monitors using the older and cheaper twistednematic (TN) technology and will also have better color stability at wide viewing angles. Other newer display technologies may also be considered if they have the appropriate gray-scale range, viewing angles, and color stability. Some nuclear medicine display software (e.g., that of MIM Software Inc.) has the ability to perform a secondary calibration to further customize the gray-scale and color tables used in the viewing program to match the monitor characteristics. If such capability is present, its use is recommended.

\section{Resolution}

Monitors should be set to their native resolution (typically, the highest resolution available in the monitor settings on your home computer). In Microsoft Windows, version 10, this resolution setting is located in your computer display settings under "scale and layout," whereas on the Apple Mac operating system it is shown under "display preferences" (Fig. 4). Likewise, resolution should also be considered in RDP application settings (Fig. 1).

\section{Color Calibration}

Consistency of color between multiple monitors and over the life of a monitor is important for semiquantitative interpretation of images. Full-screen uniform-color red, green, blue, and white images should periodically be viewed simultaneously on all monitors and evaluated visually for color consistency and uniformity. Links to such uniform-color images are available online ( 9 , also see Table 1).

Color calibration can also be achieved using a colorimeter, which often comes with calibration software. The chromaticity coordinates of a white point can be measured for absolute color accuracy or for consistency between monitors.

\section{INTERNET CONNECTION}

A reasonably high-speed internet connection is desirable. At least a $100-M b p s$ home connection is recommended, especially if large datasets need to be downloaded or if rapid scrolling or video is used as part of the interpretation. The connection speed to the internet can be assessed using publicly available tools such as DSLReports.com, Speedtest.net, and Broadbandnow.com. However, the speed to the hospital over an encrypted network connection may be severalfold slower than that measured to a public server over a nonencrypted connection. As an example, for a thin-client viewer with a 2-monitor system using Citrix or RDP connectivity to a reliable end-toend (home computer-to-server) transfer, a download speed of more than $30 \mathrm{Mbps}$ and latency of less than $150 \mathrm{~ms}$ are recommended over the encrypted connection to the hospital, but specifications will depend on multiple factors and should be reviewed with the hospital information technology department or the vendor. A wired (rather than Wi-Fi) connection to your home router is strongly recommended. Connection quality is also important since a poor-quality connection may result in frequent drops in virtual-private-network access. Connection quality is difficult to assess, but some speed test sites (e.g., DSLReports.com) provide insight into the connection quality.

Image compression will likely be used as part of a remote viewing solution. Lossless compression preserves full image quality and can be used in radiology to speed image transmission by reducing file sizes by a factor of about 2- to 3 -fold.

TABLE 1

Test Patterns

\begin{tabular}{|c|c|c|}
\hline & Web link to GIF or PNG & Downloadable DICOM image \\
\hline SMPTE test pattern & $\begin{array}{l}\text { http://gamma.wustl.edu/images/smpte.gif also available as } \\
\text { first image of https://parker.bidmc.harvard.edu/TestPatterns.html }\end{array}$ & SMPTE DICOM Image* \\
\hline Gray sphere test pattern & $\begin{array}{l}\text { http://gamma.wustl.edu/images/bwhtest.gif also available as } \\
\text { second image of https://parker.bidmc.harvard.edu/TestPatterns.html }\end{array}$ & BHW Test Pattern* \\
\hline \multirow{4}{*}{$\begin{array}{l}\text { RGB and white uniform } \\
\text { images }\end{array}$} & Red Uniform Image* & Red Uniform DICOM Image* \\
\hline & Blue Uniform Image* & Blue Uniform DICOM Image* \\
\hline & Green Uniform Image* & Green Uniform DICOM Image* \\
\hline & White Uniform Image* & White Uniform DICOM Image* \\
\hline
\end{tabular}

*Links are provided on SNMMI Physics, Instrumentation, and Data Sciences Council web page (https://www.snmmi.org/Council.aspx? ItemNumber=6565); navigate to the PIDSC Resources tab. 


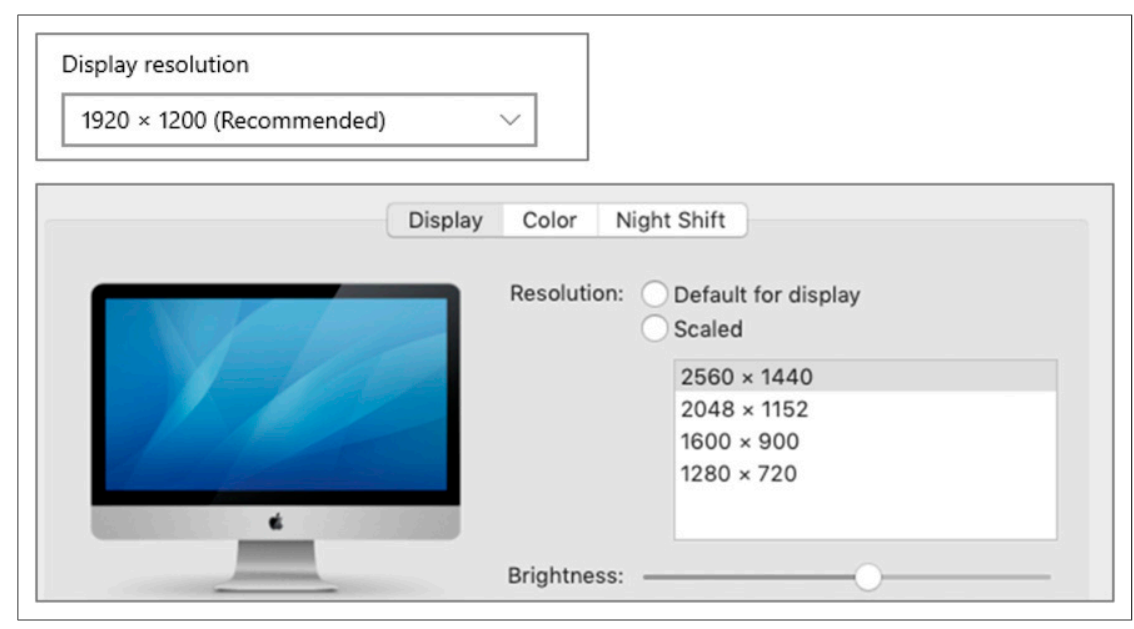

FIGURE 4. Windows (top) and Mac (bottom) screen resolution set for maximum available resolution for that monitor.

Lossy compression results in yet smaller files but induces small, irreversible changes to the image. Lossy compression of about 10 -fold has been accepted for radiology use in many imaging settings (10). Limited data are available regarding standards for lossy compression of nuclear medicine imaging, but given the lower resolution of nuclear medicine than of other imaging modalities, it is likely that standards for nuclear medicine will be no more stringent than those for other radiology fields.

If the user is reading images at home on a thin or thick client, it is likely that the compression will be handled by the viewing software and should be identical to that used in the hospital setting. If the user is viewing a remote image by first connecting to a computer at the hospital and then launching the viewing program on that computer, it is possible that the remote connection (e.g., Citrix or RDP) may impose additional image compression. Because of the uncertainty of the effects of further compression, it is recommended that any additional compression be lossless when possible. Future assessment of lossy image compression of nuclear medicine images may be useful to clarify its role in remote image viewing. Citrix compression is set by the server administrator. The ability to use lossless compression may depend on the Citrix version and the specific video cards, since compression and decompression may be offloaded to video cards for greater speed. When connecting to a centralized RDP server, you can access the settings for compression and quality under "Group Policyl ComputerlWindows Components\Remote Desktop Services\ Remote Desktop Session HostlRemote Session Environment." Any changes should be discussed with the hospital information technology department. When connecting via RDP directly to a specific desktop personal computer client, the compression settings usually cannot be modified.

The hospital information technology department may be able to perform more accurate tests and monitor network performance over prolonged intervals to diagnose connectivity issues, using tools such as iPerf (https://iperf.fr/).

\section{CONCLUSION}

The SNMMI Physics, Instrumentation, and Data Sciences Council hopes these guidelines will facilitate creation of home interpretation stations for nuclear medicine. These guidelines are based on the available literature and on a consensus of opinions rather than exhaustive tests by the council; however, they do address several key issues for at-home nuclear medicine viewing. These guidelines are designed to assist practitioners in providing appropriate care for patients. They are not inflexible rules or requirements of practice and are not intended, nor should they be used, to establish a legal standard of care. For these reasons, the SNMMI cautions against the use of these guidelines in litigation in which the clinical decisions of a practitioner are called into question. The ultimate judgment regarding the propriety of any specific procedure or course of action must be made by the physician or medical physicist in light of all the circumstances presented. Thus, there is no implication that an approach differing from the guidelines, standing alone, is below the standard of care.

\section{DISCLOSURE}

No potential conflict of interest relevant to this article was reported.

\section{REFERENCES}

1. Lunt B. Setting up a radiologist's home reporting display. MyXrayDose website. https://myxraydose.com/setting-up-a-radiologist-remote-reporting-display/. Published 2017. Accessed January 26, 2021.

2. Setting up a temporary display device for diagnostic interpretation. American Association of Physicist in Medicine website. https://w3.aapm.org/covid19/displaydevice-docx.php. Accessed January 26, 2021.

3. Song N, Zuckier LS. A dearth of specifications regarding primary diagnostic monitors (PDMs) for nuclear medicine leaves us with little guidance during the COVID-19 pandemic. J Appl Clin Med Phys. 2020;21:321-322.

4. ACR-AAPM-SIIM technical standard for electronic practice of medical imaging. American College of Radiology website. https://www.acr.org/-/media/ACR/ Files/Practice-Parameters/Elec-Practice-MedImag.pdf. Published 2007. Last revised 2017. Accessed January 26, 2021.

5. Parker JA, Christian P, Jadvar H, Sattler B, Wallis JW. The SNMMI and EANM practice guideline for tele-nuclear medicine 2.0. J Nucl Med Technol. 2014; 42:15-19.

6. Lim S-TJM, Murray M, O’Brien J. An audit of radiologist reporting room illumination levels [letter]. Radiography. 2020;27:252.

7. Luxmeter app versus measuring device: are smartphones suitable for measuring illuminance? DIAL website. https://www.dial.de/en/blog/article/luxmeter-appversus-measuring-deviceare-smartphones-suitable-for-measuring-illuminance/. Published February 23, 2016. Accessed January 26, 2021.

8. Cerqueira D, Carvalho F, Melo RB. Is it smart to use smartphones to measure illuminance for occupational health and safety purposes? In: Advances in Safety Management and Human Factors. Springer; 2017:258-268.

9. Test pattern images located on SNMMI Physics, Instrumentation, and Data Sciences Council web page (https://www.snmmi.org/Council.aspx?ItemNumber=6565), navigate to the PIDSC Resources tab. Accessed February 8, 2021.

10. European Society of Radiology. Usability of irreversible image compression in radiological imaging: a position paper by the European Society of Radiology (ESR). Insights Imaging. 2011;2:103-115. 